\title{
Optimization and Permeation Study of Novel Topically Applied Antilipemic Lotion Using Central Composite Design
}

\author{
Asma Aslam ${ }^{1}$, Rummana Riaz ${ }^{1}$, Yasser Shahzad ${ }^{2}$, Atif Akber ${ }^{3}$, Ikram Ullah Khan ${ }^{4, *}$, Syed Nisar Hussain Shah ${ }^{5}$ \\ ${ }^{1}$ Multan College of Pharmacy and Management Sciences, Pakistan \\ ${ }^{2}$ Centre of Excellence for Pharmaceutical Sciences, North-West University, South Africa \\ ${ }^{3}$ Department of Statistics, Bahauddin Zakariya University, Multan, Pakistan \\ ${ }^{4}$ College of Pharmacy, Government College University, Faisalabad, Pakistan \\ ${ }^{5}$ Faculty of Pharmacy, Bahauddin Zakariya University, Multan, Pakistan \\ *Corresponding author: ikramglt@gmail.com
}

Received September 20, 2014; Revised September 27, 2014; Accepted September 30, 2014

\begin{abstract}
In this study, synergistic effect of penetration enhancers, namely propylene glycol (PG) and sodium lauryl Sulphate (SLS) on transdermal absorption of 2-Methyl-2-phenoxy-propionic acid (MPA) was evaluated with aid of response surface methodology (RSM) based formulations. The prepared formulations were characterised and subsequently subjected to diffusion cell experiments through a well-established skin mimic, namely silicone membrane. Data obtained were statistically analysed using analysis of variance (ANOVA) and regression analysis. Comparisons were made with the control and among different parameters of formulations. PG (X1) and SLS (X2) were selected as independent variables, whereas the cumulative amount of MPA were used to calculate dependent permeation kinetic parameters like lag time ( $\mathrm{l}_{\mathrm{lag}}$ ) and enhancement ratios (ER). It was observed from the results that PG had a significant effect on MPA absorption from the lotion followed by SLS and their combination showed a significant enhancement $(\mathrm{p}<0.05)$ in the flux from the formulated lotions compared to the control. The highest enhancement ratio $(\mathrm{ER}=8.6)$ was observed for $\mathrm{L} 4$. Contour plots were then drawn to depict the relationship between independent and response variables. Among the formulations, L4 showed highest flux value with less lag time (12.2 $\mathrm{min}$ ), thereby, identified and selected as an optimized formulation. In conclusion, the combination of PG and SLS can be successfully utilized as permeation enhancers for transdermal drug delivery of MPA with minimal side effects.
\end{abstract}

Keywords: propylene glycol, sodium lauryl sulphate, permeation, response surface methodology, lotion

Cite This Article: Asma Aslam, Rummana Riaz, Yasser Shahzad, Atif Akber, Ikram Ullah Khan, and Syed Nisar Hussain Shah, "Optimization and Permeation Study of Novel Topically Applied Antilipemic Lotion Using Central Composite Design.” American Journal of Pharmacological Sciences, vol. 2, no. 5A (2014): 8-14. doi: 10.12691/ajps-2-5A-2.

\section{Introduction}

2-methyl-2-phenoxy-propionic acid (MPA) (Figure 1) is a drug from the novel series of potent HDLc raising agents which plays a key role in lipid homeostasis [1]. It is used as hypocholesterolemic and hypolipemic (as antilipemic) inducing agent [2] and to reduce numbers of colon and rectum polyps in patients with familial adenomatous polyposis [3]. It is also used as uricosuric inducing agents. Its derivatives, for instance fenofibrate, are typically administrable in the preventive or curative treatment of gout and cardiovascular diseases [4].

Transdermal drug delivery involves maintaining constant drug plasma levels to improve patient compliance and the amount of drug available at targeting site is lower than that of the oral route, but the absorbed dose appears to be sufficient for therapeutic use without inducing any side-effects [5]. Preferably, intact drug should penetrate through the skin to the underlying blood supply without any drug accumulation in the layers of the skin. Transdermal delivery systems are considered to be a control release dosage forms and it should be scientifically supported for in vivo and in vitro claims of controlled release features and therefore have to be approved based on clinical safety and efficacy studies [6,7]. Chemical permeation enhancers facilitate the absorption of permeant through the skin by temporarily decreasing the impermeability of the skin [2]. Penetration of drugs into and through the skin involves passive diffusion which occurs very slowly and is the only means to transfer drugs across the skin [8]. Lipid soluble drugs can readily pass through the intercellular lipid bilayers of the cell membranes whereas water soluble drugs are able to pass through the skin because of the hydrated intracellular proteins [5]. Combination of enhancers can enhance the drug penetration synergistically and limits the potential 
toxicity associated with the use of a high concentration of permeation enhancers when used alone, thus, ensuring safety and efficacy $[9,10,11]$. The ability of a chemical to breach the skin barrier is dependent on its physicochemical properties and the interactions with the skin [12,13]. PG is a water soluble and viscous liquid containing two hydroxyl groups and in recent years it has been proved to be a promising permeation enhancer widely used for transdermal drug delivery systems in pharmaceutical and cosmetic industry [14]. SLS is soluble and compatible with many organic solvents including alcohol and PG. SLS has been used as a permeation enhancer for a wide variety of drugs such as steroids, antibiotics and antiviral agents and effective at low concentrations between $0.1-5 \%$ [12] as it partitions into the lipid bilayer and disrupts their packing. Studies have shown that PG and SLS have been efficiently used as permeation enhancers [15].

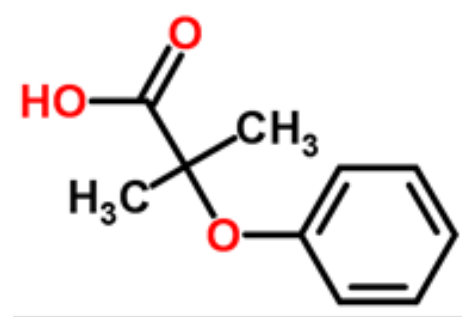

Figure 1. Chemical structure of 2-methyl-2-phenoxy-propionic acid (MPA)

Here we report optimization and assessment of the feasibility of MPA being used in the form of a topically applied lotion formulation and to analyze the synergistic effect of PG and SLS as permeation enhancers in the formulated hydro-alcoholic lotions of MPA.

\section{Materials and Methods}

\subsection{Materials}

2-Methyl-2-phenoxy-propionic acid was a kind gift from Zakfas Pharma Pvt. Ltd., Pakistan. Other chemiclas used were: propylene glycol (Merck, Germany), sodium lauryl sulfate (Fluka, Germany), isopropyl alcohol (Merck, Germany), Tween-20 (Merck, Germany ), sodium chloride (Merck, Germany), potassium chloride (Aldrich Chemical), potassium dihydrogen phosphate (Fluka, Germany), disodium hydrogen phosphate (Fluka, Germany), methanol (Merck, Germany), vacuum Grease (Dow Corning USA), Carbomer-980 (Merck, Germany), Sodium hydroxide (Shama Laboratory chemical works Lahore-Pakistan). Double distilled water was used throughout the study. All these chemicals were used as received with a minimum purity of $99 \%$.

\subsection{Methods}

\subsubsection{High-performance Liquid Chromatography (HPLC) Analysis}

Quantitative analysis of MPA was performed as described previously [16] using a Waters HPLC system (Elstree, UK) equipped with a 600E pump, a 484 UVvisible detector, an auto-sampler and a C-18 Nucleosil ${ }^{\circledR} 5$ $\mu \mathrm{m}$ column of $150 \mathrm{~mm}$ length and $4.5 \mathrm{~mm}$ internal diameter (Alltech Associates, Deerfield IL). The mobile phase consisted of acetonitrile-methanol-15 mM potassium di-hydrogen phosphate buffer, pH 7.3 (30:5:65 v/v). Mobile phase was filtered through $0.45 \mu \mathrm{m}$ filter and degassed using ultrasonic bath for 30 min prior to use. The flow rate was adjusted to $1 \mathrm{~mL} / \mathrm{min}$ and UV detector was set at $230 \mathrm{~nm}$ wavelength. The HPLC analysis was performed at ambient temperature.

\subsubsection{Solubility and Partition Coefficient Determination}

Solubility studies were conducted in various solvents: methanol, distilled water and mixture of methanol and phosphate buffered saline $(1: 1 \mathrm{v} / \mathrm{v})$. Excess amount of pure MPA was added in separate $50 \mathrm{~mL}$ conical flasks containing $10 \mathrm{~mL}$ of each solvent. These flasks were sonicated for an hour, and agitated in a thermostatically controlled shaker, with a constant temperature of $37 \pm 1^{\circ} \mathrm{C}$ for $48 \mathrm{~h}$. The suspensions were then centrifuged at 2000 rpm for 30 minutes and supernatant aliquot was taken out by a pipette and analyzed through HPLC to determine the concentration in $\mathrm{mg} / \mathrm{mL}$.

For partition coefficient determination, $10 \mathrm{mg}$ of MPA was dissolved in a pre-equilibrated mixture of $0.1 \mathrm{~N}$ sodium hydroxide and octanol in a separating funnel. This was shacked vigorously for 10 minutes and left for each layer to settle down for about $12 \mathrm{~h}$. Then separated layers were collected in test tubes and analyzed by HPLC to calculate the octanol and water $(0.1 \mathrm{~N}$ sodium hydroxide was considered as aqueous phase) partition coefficient $(\mathrm{Ko} / \mathrm{w})$. Each experiment was carried out in triplicate $(n=3)$.

\subsubsection{Preparation of Topical Lotion}

In order to optimize the formulated lotions and estimation of the influence of formulation variables on MPA permeation, a central composite design (CCD) with $\alpha=2$ was employed as per standard protocol. The factors, namely PG (X1) and SLS (X2) planned at 5 levels $(-2,-1$, $0,1,2)$ were selected based on the results of primary experiments. Preliminary experiments were conducted employing PG and SLS combination at two concentrations, namely $3 \%$ and $5 \%$, respectively. It was found that the combination of enhancers could enhance the permeant. Consequently, it was decided to optimize formulated lotions within the studied range. The central point $(0,0)$ was studied in quintuplicate. Rest of the formulations ingredients were kept invariant throughout the study as given in Table 1. MPA hydro-alcoholic lotions $(100 \mathrm{~mL}$ each) were prepared as per the CCD design. Essentially, 1 g MPA was dissolved in $20 \mathrm{ml}$ of mixture of PBSmethanol $(1: 1 \mathrm{v} / \mathrm{v})$ followed by the addition of PG and SLS according to the CCD design. It was stirred over a magnetic stirrer for $30 \mathrm{~min}$ until solution was homogenized. Isopropyl alcohol $(20 \mathrm{~mL})$ was taken in a separate flask and $0.2 \mathrm{~g}$ carbopol-980 was added to it with constant stirring. To this solution, $4 \mathrm{~mL}$ Tween-20 was added and it was stirred for another $30 \mathrm{~min}$. Both solutions were then mixed over continuous stirring and final volume (100 mL) was achieved by adding mixture of PBS and methanol (1:1 v/v). An enhancer (PG and SLS) free lotion was also prepared as control (LC).

\subsection{In vitro Characterization}




\subsubsection{Physical Appearance, $\mathrm{pH}$ and Viscosity Determination}

The physical appearance included visual inspection for clarity and precipitation of the prepared lotions. The formulated lotions were assessed for $\mathrm{pH}$ value at $25 \pm$ $0.5^{\circ} \mathrm{C}$ using a digital $\mathrm{pH}$ meter (WTW, pH 526 Germany). Viscosity $(\eta)$ measurements were carried out at room temperature $\left(25 \pm 0.5^{\circ} \mathrm{C}\right)$ using a Brookfield viscometer (Model RVTDV II, Brookfield Engineering Laboratories, Stoughton, MA).

\subsubsection{Stability Studies}

As with tables and equations, figures should be set in one column if possible unless two-column display is essential. The resolution of graphics and image should be adequate to reveal the important detail in the figure.

\subsection{Ex Vivo Studies}

\subsubsection{Permeation across Skin Mimic}

Permeation studies across an established skin mimic, namely silicone membrane were performed using Franztype diffusion cells that have a receptor phase of $\sim 5 \mathrm{ml}$, and a diffusional area of $0.788 \mathrm{~cm}^{2}$. Sheets of silicone membrane were cut to appropriate sizes in round-shape and soaked overnight in the receptor phase (mixture of PBS and methanol; $1: 1 \mathrm{v} / \mathrm{v}$ ). The membrane was then placed between the two compartments of the diffusion cells. Vacuum grease was used to produce a leak-proof seal between the membrane and the two compartments of the diffusion cells. The receptor compartment was filled with pre-sonicated receptor phase to ensure no air bubbles build-up in the receptor compartment. Experiments were performed in occluded condition to prevent evaporation of donor and receptor solution.

Uniform mixing of the receptor phase was obtained with a magnetic stirrer that was placed in the receptor compartment. The diffusion cells were placed on a stirring bed immersed in a water bath at $37\left( \pm 1^{\circ} \mathrm{C}\right)$, to maintain a temperature of $\sim 36^{\circ} \mathrm{C}$ at the membrane surface. After 1 hour the receptor phase was completely removed and refilled with fresh pre-thermostated receptor solution.

The donor compartment was charged with $1 \mathrm{~mL}$ of hydro-alcoholic lotion. After elapsed time of 15, 30, 45, $60,90,120$, and 180 minutes interval, $0.2 \mathrm{ml}$ of sample from receptor solution was drawn using micro pipette, followed by addition of same volume of fresh pre thermostated receptor solution to maintain the sink conditions. Withdrawn solutions were assayed immediately by HPLC for MPA quantification. Experiments were conducted in triplicate to obtain a statistically significant data.

\subsubsection{Draize's Skin Irritation Test}

Primary test for irritation was performed on human volunteers after selecting optimized formulation. Eleven volunteers (both male and female) of age $21 \pm 2$ were selected and small amount of formulated lotion was applied on an area of 2 square inch to the back of hand. The volunteers were observed for lesions or irritation.

\subsubsection{Sensatory Perception Panel Test}

A questionnaire containing six questions was prepared and given to each volunteer (total of eleven volunteers both male and female of age $21 \pm 2$ ) for sensory evaluation of the optimized lotion and average points were calculated from the points assigned (nine values from -4 to +4 indicating very bad to excellent respectively) by each volunteer for each question.

\subsection{Data Analysis}

Fick's second law of diffusion, the cumulative amount of drug (Qt) appearing in the receptor solution in time $t$ is expressed in Eq. 1:

$$
Q_{t}=A K L C_{0}\left[\begin{array}{l}
\left(\frac{D t}{L^{2}}\right)-\left(\frac{1}{6}\right) \\
-\left(\frac{2}{\pi^{2}}\right) \sum \frac{(-1)^{n}}{n^{2}}
\end{array}\right] \times \exp \left(\frac{D^{n} 2 \pi^{2} t}{L^{2}}\right)
$$

where $\mathrm{A}$, is the effective diffusion area, $\mathrm{C}$, represents the drug concentration which remains constant in the vehicle, $\mathrm{D}$ is the diffusion coefficient, $\mathrm{L}$ denotes the thickness of the membrane and $\mathrm{K}$ is the partition coefficient of the drug between membrane and vehicle. At steady state, it is expressed in Eq. 2:

$$
\frac{Q_{t}}{A}=K L C_{0}\left[\left(\frac{D t}{L^{2}}\right)-\left(\frac{1}{6}\right)\right]
$$

The steady state flux ( $\mathrm{J}$ ) was calculated from the slope of the linear plot of the cumulative amount permeated per unit area as a function of time, in the steady-state region where the drug would pass by constant rate. The lag time was determined from the $\mathrm{x}$-intercept of the slope at the steady state. The flux is expressed in Eq. 3:

$$
J=\frac{C_{0} K D}{L}=C_{0} K_{P}
$$

From this relation the permeability coefficient was calculated using Eq. 4:

$$
K_{P}=\frac{J}{C_{0}}
$$

The effectiveness of penetration enhancers (enhancement ratio, ER) was calculated from the ratio of MPA flux in the presence and absence of enhancers.

The analysis of responses, namely lag time $\left(\mathrm{t}_{\mathrm{lag}}\right)$ and enhancing ratio (ER) were performed using Design Expert software version 7. Linear, quadratic and cubic mathematical models were employed. The best fit model was selected based on the comparison of several parameters including the multiple correlation coefficients (R2), adjusted multiple correlation coefficients (adjusted R2), predicted residual sum of square (PRESS), and the lack of fit (p-value). Experimental design resulted in a quadratic polynomial equation which is expressed in Eq.6:

$$
\begin{aligned}
\mathrm{Y}= & \beta 0+\beta 1 \mathrm{X} 1+\beta 2 \mathrm{X} 2+\beta 12 \mathrm{X} 1 \mathrm{X} 2 \\
& -\beta 12 \mathrm{X} 12-\beta 22 \mathrm{X} 22
\end{aligned}
$$

Where $\mathrm{Y}$ is the dependent variable (response), $\beta 0$ is a constant representing the mean of the dependent variable obtained in each experiment; X1 and X2 are the independent variables; $\mathrm{X} 1 \mathrm{X} 2$ are the interaction terms; $\mathrm{X} 12$ and $\mathrm{X} 22$ are the quadratic term and $\beta 1, \beta 2$ are the coefficients. This expression provides an explanation of 
effect of the different independent variables. A positive sign of coefficient indicates a synergistic effect whereas a negative term indicates an antagonistic effect upon the response. Large coefficient means the causal factor has potent influence on the response. Afterwards contour and 3D-surface plots visualizing the simultaneous effect of the causal factors on the response were established [18]. Oneway ANOVA was applied to estimate the significance of the model $(p<0.05)$. All measured data are expressed as mean \pm standard deviation (S.D.). Each measurement was executed in three replicates $(n=3)$.

\section{Results}

\subsection{Solubility and Partition Coefficient}

The solubility study done in water was $0.58 \pm 0.01$ $\mathrm{mg} / \mathrm{mL}$; in methanol $68.29 \pm 0.01 \mathrm{mg} / \mathrm{mL}$; in PBS $66.52 \pm$ $0.02 \mathrm{mg} / \mathrm{mL}$ and in mixture of PBS with methanol $78.71 \pm$ $0.14 \mathrm{mg} / \mathrm{mL}$. Solubility of MPA in PBS-methanol mixture
(1:1 v/v) was 135-fold higher which might be due to the solvent polarity difference between two different solvent systems, namely water and PBS-methanol mixture. The value of Ko/w determined was $1.13 \pm 0.20$.

\subsection{In vitro Characterization Data}

In vitro characterization includes appearance and other physical properties like clarity, precipitation and homogeneity, $\mathrm{pH}$ and viscosity. Upon preparation all the formulated lotions appeared transparent and aggregate free homogeneous solutions. All the lotions exhibited a $\mathrm{pH}$ value from 4.04 to 4.43 with no significant differences ( $\mathrm{p}>$ 0.05 ) existing between each formulation (data not shown). Moreover, an insignificant difference $(p>0.05)$ in lotion viscosities (Table 1) with respect to PG and SLS content were observed among formulations.

With respect to stability testing, all the prepared formulations were stable over 6 months accelerated stability testing period with drug content ranging from 98.31-99.81\% (Table 1).

Table 1. Permeation kinetic parameters, viscosity and drug content of formulated lotions with RSM (mean \pm S.D; n=5)

\begin{tabular}{|c|c|c|c|c|c|c|c|c|c|c|}
\hline \multirow{2}{*}{ Formulations } & \multicolumn{2}{|c|}{ Coded factors } & \multicolumn{2}{|c|}{ Actual factor (g) } & \multicolumn{2}{|c|}{ Response values } & \multirow{2}{*}{$J\left(\mu \mathrm{g} / \mathrm{cm}^{2} . \mathrm{min}\right)$} & \multirow{2}{*}{$\begin{array}{c}D \times 10^{-2} \\
\left(\mathrm{~cm}^{2} / \mathrm{min}\right)\end{array}$} & \multirow{2}{*}{$\begin{array}{c}\eta \times 10^{-2} \\
\text { (dynes.s/cm²) }\end{array}$} & \multirow{2}{*}{$\begin{array}{l}\text { Drug content } \\
\text { (\%) }\end{array}$} \\
\hline & $\mathrm{X}_{1}$ & $\mathrm{X}_{2}$ & $\mathrm{X}_{1}: \mathrm{PG}$ & $\mathrm{X}_{2}: \mathrm{SLS}$ & $\mathrm{Y}_{1}: \mathrm{t}_{\mathrm{lag}}$ & $\mathrm{Y}_{2}: E R$ & & & & \\
\hline L1 & 0 & 2 & 2.6 & 3.1 & 12.4 & 5.8 & $0.17 \pm 0.02$ & $1.60 \pm 0.53$ & 89 & 99.89 \\
\hline L2 & -1 & -1 & 1.9 & 1.0 & 13.2 & 6.6 & $0.20 \pm 0.03$ & $1.70 \pm 0.85$ & 91 & 98.76 \\
\hline L3 & 0 & 0 & 2.6 & 1.7 & 12.9 & 7.9 & $0.24 \pm 0.06$ & $1.66 \pm 0.79$ & 90 & 98.58 \\
\hline L4 & 0 & -2 & 2.6 & 0.3 & 12.2 & 8.6 & $0.26 \pm 0.07$ & $1.57 \pm 0.83$ & 92 & 98.97 \\
\hline L5 & -1 & 1 & 1.9 & 2.4 & 5.5 & 3.4 & $0.10 \pm 0.04$ & $0.71 \pm 0.18$ & 91 & 99.78 \\
\hline L6 & 1 & -1 & 3.3 & 1.0 & 7.9 & 4.3 & $0.13 \pm 0.01$ & $1.02 \pm 0.87$ & 90 & 99.43 \\
\hline L7 & 1 & 1 & 3.3 & 2.4 & 7.6 & 3.3 & $0.10 \pm 0.01$ & $0.98 \pm 0.24$ & 90 & 98.76 \\
\hline L8 & 0 & 0 & 2.6 & 1.7 & 13.7 & 7.5 & $0.23 \pm 0.02$ & $1.77 \pm 0.93$ & 92 & 98.31 \\
\hline L9 & 2 & 0 & 4.0 & 1.7 & 7.53 & 4.6 & $0.14 \pm 0.08$ & $0.97 \pm 0.31$ & 89 & 98.56 \\
\hline L10 & 0 & 0 & 2.6 & 1.7 & 13.3 & 8.0 & $0.24 \pm 0.04$ & $1.72 \pm 0.96$ & 89 & 99.11 \\
\hline L11 & -2 & 0 & 1.2 & 1.7 & 6.87 & 4.9 & $0.15 \pm 0.03$ & $0.89 \pm 0.21$ & 94 & 98.92 \\
\hline L12 & 0 & 0 & 2.6 & 1.7 & 16.4 & 7.1 & $0.21 \pm 0.07$ & $2.12 \pm 1.03$ & 90 & 98.45 \\
\hline L13 & 0 & 0 & 2.6 & 1.7 & 16.3 & 7.2 & $0.22 \pm 0.08$ & $2.10 \pm 1.19$ & 91 & 98.67 \\
\hline
\end{tabular}

\subsection{Diffusion Studies}

The in vitro permeation study was done by using hydroalcoholic lotions of MPA in the donor compartment of the modified Franz diffusion cell. The cumulative amount that has passed through silicone membrane was estimated by HPLC. Figure 2 elucidated the cumulative amount of drug permeated as a function of time from lotion formulations as per CCD. It can be seen from the Figure 2 also explained that the highest permeation was achieved for L9 that contained equal coded level (amount of PG and SLS at medium level). The steady state flux was calculated by a linear regression between cumulative amount permeated and time. The lag time ( $\left.\mathrm{t}_{\text {lag }}\right)$, which is directly related to the drug diffusivity, was calculated from the $\mathrm{x}$-intercept of the cumulative amount of drug permeated as a function of time. The permeation parameters are listed in Table 1. The lag time $\left(\mathrm{t}_{\text {lag }}\right)$ values for lotion formulations ranged from $5.50 \mathrm{~min}$ to $16.40 \mathrm{~min}$, where optimized lotion, L4 has $12.20 \mathrm{~min}$. The steady state flux (J) ranged from $0.101 \pm$ 1.32 to $0.26 \pm .26$, and enhancement ratio (ER) ranged from 3.27 to 8.60 for lotion formulations, which indicated that the permeation of MPA from its lotion formulation was significantly influenced by the proportion of the formulation variables, namely PG and SLS while lag time and ER values for enhancer containing lotions were significantly different $(\mathrm{p}<0.05)$ from that of the control (LC).

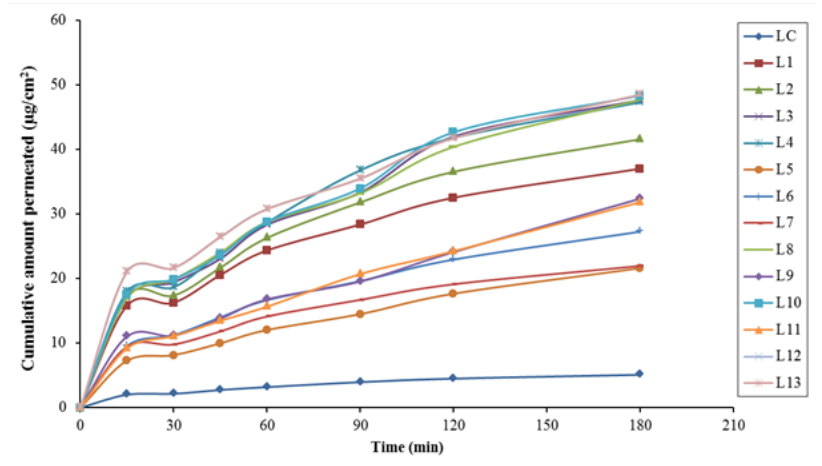

Figure 2. Cumulative amount of formulated lotions permeated through silicone membrane

The effect of PG and SLS on the permeation of MPA from its lotion formulations was quantified by analysing the responses ( $t_{\text {lag }}$ and ER) using RSM. The estimation of quantitative effects of the factor combination and their levels on responses was carried out by fitting data to linear, quadratic and cubic models. The best fit model was quadratic which could be represented as: 


$$
\begin{aligned}
\mathrm{Y} 1\left(\mathrm{t}_{\text {lag }}\right)= & 13.531-0.1566 \mathrm{X} 10.633 \mathrm{X} 2+1.850 \mathrm{X} 1 \mathrm{X} 2 \\
& +1.892 \mathrm{X} 12+0.617 \mathrm{X} 22 \\
\mathrm{Y} 2(\mathrm{ER})= & 6.890-0.250 \mathrm{X} 1-0.817 \mathrm{X} 2+0.550 \mathrm{X} 1 \mathrm{X} 2 \\
& +0.738 \mathrm{X} 12+0.126 \mathrm{X} 22
\end{aligned}
$$

The significance of formulation variables on MPA permeation was evaluated through multiple linear regression analysis (MRA) using Design Expert version 7. The comparative values of R2, adjusted R2, PRESS, lack of fit (p-value) are summarized in Table 2.

Table 2. Summarized statistical parameters of each response variable determined by MRA

\begin{tabular}{|c|c|c|}
\hline \multirow{2}{*}{ Regression Coefficients } & \multicolumn{2}{|c|}{ Coefficient estimates } \\
\cline { 2 - 3 } & $\mathrm{t}_{\mathrm{lag}}$ & $\mathrm{ER}$ \\
\hline$\beta_{0}$ & 13.53 & 6.89 \\
$\beta_{1}\left(\mathrm{X}_{1}\right) \mathrm{PG}$ & -0.16 & -0.25 \\
$\beta_{2}\left(\mathrm{X}_{2}\right) \mathrm{SLS}$ & -0.63 & -0.82 \\
$\beta_{12}\left(\mathrm{X}_{1} \mathrm{X}_{2}\right)$ & 1.85 & 0.55 \\
$\beta_{1}{ }^{2}\left(\mathrm{X}_{1}{ }^{2}\right)$ & -1.89 & -0.74 \\
$\beta_{2}{ }^{2}\left(\mathrm{X}_{2}{ }^{2}\right)$ & -0.62 & -0.13 \\
Model $(p$-value) & 2.33 & 1.86 \\
$\mathrm{R}^{2}$ & 0.62 & 0.57 \\
Adjusted $\mathrm{R}^{2}$ & 0.36 & 0.26 \\
PRESS & 397.81 & 116.26 \\
C.V\% & 26.24 & 25.61 \\
Lack of Fit (p-value) & 5.70 & 33.52 \\
\hline
\end{tabular}

\subsection{Skin Irritation and Panel Test}

Draize's skin irritation test revealed that optimized lotion (L4) did not show any signs of irritation in terms of erythema, oedema, redness, ulceration and haemorrhaging.

Sensatory perception testing involving a panel of volunteers rated revealed high acceptability for the optimized MPA lotion. The panel scored the lotion between 2.5 and 3.8 in terms of all categories: ease of application, skin sensation immediately after application, long-term skin sensation, skin 'shine' and induced skin softness.

\section{Discussion}

PG and SLS combination was employed in this study as permeation enhancers. As far as we could ascertain, there is no published report describing the effect of PG and SLS combination on transdermal absorption of MPA. PG can enhance the solubility of drug in the membrane. Furthermore, PG as a hydrophilic material with two hydroxyl group could replace water at the binding sites in the polar head group region and may act in a similar way to water in enhancing the drug permeation [19]. SLS, an anionic surfactant is being used in various cleaning and hygiene products, however, precise sensitivity (irritation) of the human face to care products urged to study irritation caused by SLS in various regions of human face [20]. From this perspective our study did not show any irritation effect on human volunteers. Reduction in drug transport rate through membrane in the presence of enhancers may be attributed to the ability of surfactants to form micelles. These surfactant micelles solubilize drug species which decreases the thermodynamic activity of the drug, thus, decreasing the driving force of the drug absorption. Moreover, it may be expected that SLS penetrate slowly which may cause a reduction in percutaneous enhancing properties [15].

Analysis of RSM data elucidated a significant model probability at p-value less than 0.05 and insignificant lack of fit at p-value greater than 0.05 , implying a possible relationship between the factors and responses. The main influence of $\mathrm{X} 1$ and $\mathrm{X} 2$ shows the average result of changing one variable at a time from its low to higher level while interaction effects of X1, X2, X12 and X22 represent the results when both factors were altered simultaneously. It was observed that interaction of factors (X1X2) influenced $t_{\text {lag }}$ and ER relatively higher than the main influence indicating the PG and SLS combination was more suitable in enhancing permeation of MPA. The negative coefficients of X12 and X22 imply an unfavourable effect of the factors on the permeation of drug form the formulation (Table 2). The R2 values for Eq. 7 and 8 were found to be 0.62 and 0.57 , respectively, indicating a reasonable correlation coefficient of the fitted model. The lag time values (Table 1) explicated a significant difference between the formulations based on axial points of the CCD. The lag time is dependent on the rate at which drug diffuses through the skin/membrane, thus a higher drug diffusivity leads to reduction in lag time [21]. The longest lag times were obtained for the formulations carrying medium levels of PG (2.6 g in L1, L3, L4, L8, L10, L12, L13). This may be explained on the basis that the enhancing effect of PG is exerted by enhancing the drug partitioning into membrane. To do this, PG has to partition into the membrane, thus changing its solubilizing power with subsequent increased drug partition into the membrane [22]. The flux values for L3, L8, L10, L12 and L13 were found to be similar (Table 1) as these lotion formulations were based on central points $(0,0)$ of the CCD and have similar levels of PG and SLS. It should be noted that lotion formulation having equal amount of PG and SLS (formulations based on central points and L3, L8, L10, L12 and L13) showed a gradual increase in the permeation profile with respect to factor level with L4 showing the highest flux $(0.26 \pm 0.07$ $\mu \mathrm{g} / \mathrm{cm} 2 . \mathrm{min}$ ) as given in Table 1. The observed increase in the permeation profile of MPA, when enhancer combination was used in equal volume, could be attributed to the fact that PG dehydrates and desolvate the membrane [19] and disrupts the matrix complex with subsequent increased solubility of MPA in this membrane. Additionally, presence of SLS also contributed to increase in the solubility of MPA and may lose its retarding ability in the presence of PG which has disrupted the matrix complex in the membrane [23]. The retarding effect of SLS is owing to its ability to form a surfactant layer on the silicone membrane [24]. Since PG tends to disturb the matrix complex in the membrane, it may be assumed that SLS may not act as permeation retardant when membrane is disrupted by co-enhancer. This resulted in an increased amount of solubilized MPA in the membrane that creates a concentration gradient which facilitated drug permeation through the membrane.

This was further analysed by constructing contour and 3-D surface plots (Figure 3 and Figure 4) which are useful in visual explanation of the effect of factors on responses. 
From Figure 3a \& 3b, it can be seen that $t_{\text {lag }}$ was fluctuating with increase/decrease of the concentration of PG/SLS in the formulations. A significant increase in lag time (12.4 - 16.4 min)was observed when medium levels of PG (2.6 g) and SLS (1.7 g) were used while a significant decrease in lag time were observed when low level of PG (1.9 g) and high level of SLS (1.7 g) were

$\underline{3 a}$
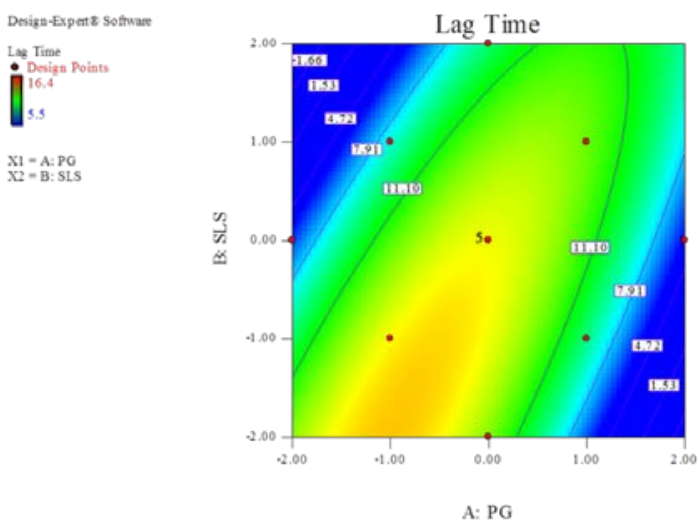

used. Figure 4a \& 4b revealed that medium level of PG in the formulation has positive effect on the ER while SLS levels did not show any considerable influence; in fact a negative influence can be observed based on regression analysis. However, a gradual increase in the permeation rate of MPA was observed with medium levels of PG and SLS combination in the formulations.

$\underline{3 \mathbf{b}}$

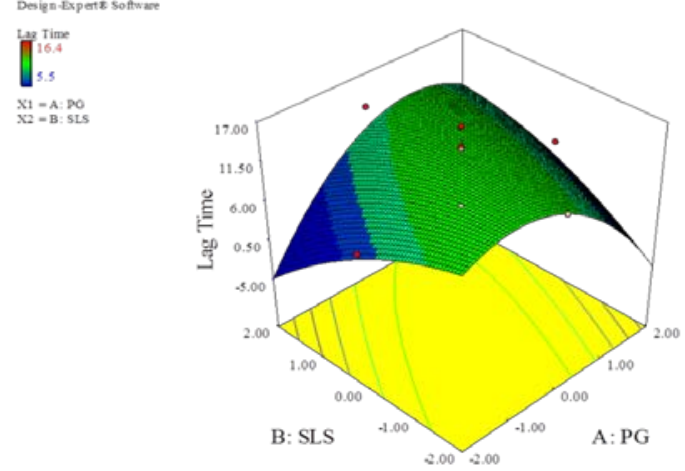

Figure 3. Estimated contour plot (3a) and response surface (3b), elucidating the relationship between the permeation enhancers and the lag time

$\underline{4 a}$

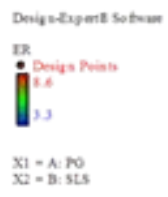

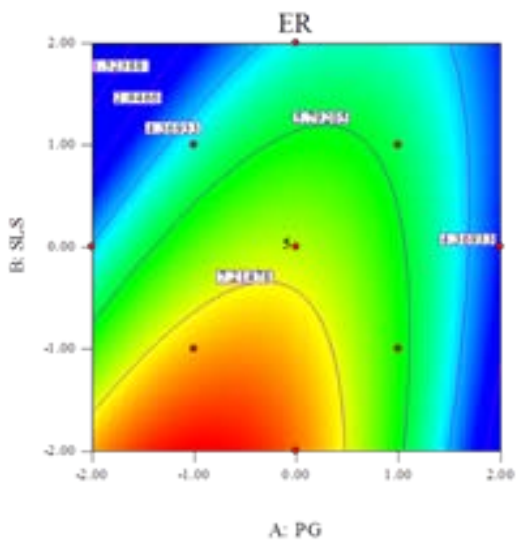

$\underline{4 b}$

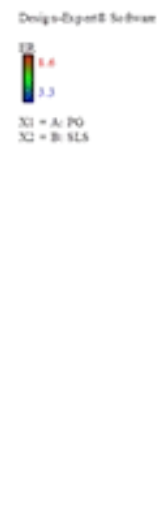

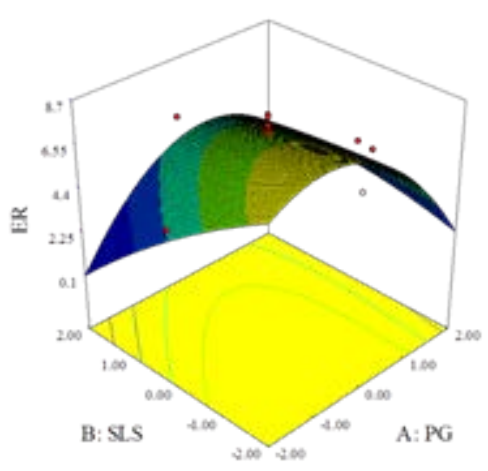

Figure 4. Estimated contour plot (4a) and response surface (4b), elucidating the relationship between the permeation enhancers and ER

The optimized formulation (L4) has proved to be safe in terms of producing any irritation to the skin as revealed by the Draize's skin irritation test, and has passed the panel test involving human volunteers.

\section{Conclusions}

The result of this study shows that RSM is a useful statistical tool to study the effect of the selected variables on the chosen responses ( $t_{\text {lag }}$ and ER). It is clear that the individual characteristics of the MPA lotion formulations were significantly affected by the formulation factors and their interactions. The permeation capacity was significantly increased and the lag time $\left(\mathrm{t}_{\mathrm{lag}}\right)$ was higher by using PG and SLS combination at medium level. However, appropriate balancing between various combinations of the two dependent factors was imperative to acquire highest permeation of MPA. Among various combinations of the PG and SLS used in this study, a combination of both PG and SLS in L4 could be used for the design of suitable MPA TDDS. There are number of other options available i.e. inclusions of some other permeation enhancers or increase in the percentage of one or the two of the permeation enhancer. Further work is needed in order to achieve clinical levels in experimental animals and humans.

\section{Acknowledgements}

The authors are thankful to Faculty of Pharmacy, Bahauddin Zakariya University, Multan for supporting this research work and also M/S Zakfas (Pvt) Ltd. for facilitating and extending their co-operation.

\section{References}

[1] Sierra M.L., Beneton V., Boullay A-B., Boyer T., Brewster A.G., Donche F. Substituted 2-[(4-aminomethyl) phenoxy]-2methylpropionic acid PPAR $\alpha$ agonists. 1. Discovery of a novel 
series of potent HDLc raising agents. Journal of medicinal chemistry, 50, 685-695, 2007.

[2] Kennard C.H.L., Smith G., White A. Structural aspects of phenoxyalkanoic acids. The structures of phenoxyacetic acid,(+-)2-phenoxypropionic acid,(+-)-2-(4-chlorophenoxy) propionic acid, 2-methyl-2-phenoxypropionic acid and 2-(4-chlorophenoxy)-2methylpropionic acid. Acta Crystallographica Section B: Structural Crystallography and Crystal Chemistry, 38, 868-875, 1982.

[3] Thompson J.P., Christopher M.M., Ellison G.W., Homer B.L., Buchanan B.A. Paraneoplastic leukocytosis associated with a rectal adenomatous polyp in a dog. Journal of the American Veterinary Medical Association, 201, 737-738, 1992.

[4] Mokale S.N., Elgire R., Sakle N., Shinde D.B. Synthesis, hypolipidemic and hypoglycemic activity of some novel 2-(4-(2substituted aminothiazole-4-yl) phenoxy)-2-methyl propanoic acid derivatives. Bioorganic \& medicinal chemistry letters, 21, 682685, 2011.

[5] Prausnitz M.R., Langer R. Transdermal drug delivery. Nature Biotechnology, 26, 1261-1268, 2008.

[6] Guy R.H., Hadgraft J. Transdermal drug delivery: A simplified pharmacokinetic approach. International Journal of Pharmaceutics, 24, 267-274, 1985.

[7] Alexander A., Dwivedi S., Ajazuddin., Giri T.K., Saraf S., Saraf S. Approaches for breaking the barriers of drug permeation through transdermal drug delivery. Journal of Controlled Release, 164, 2640, 2012.

[8] Cooperberg B.A., Cryer P.E. Insulin reciprocally regulates glucagon secretion in humans. Diabetes, 59, 2936-2940, 2010.

[9] Fox L.T., Gerber M., Du Plessis J., Hamman J.H. Transdermal drug delivery enhancement by compounds of natural origin. Molecules, 16, 10507-10540, 2011.

[10] Shahzad Y., Khan Q., Hussain T., Shah S.N.H. - Influence of cellulose derivative and ethylene glycol on optimization of lornoxicam transdermal formulation. International Journal of Biological Macromolecules, 61, 26-32, 2013.

[11] Shah S.N.H., Tahir M.A., Safdar A., Riaz R., Shahzad Y., Rabbani M. Effect of permeation enhancers on the release behavior and permeation kinetics of novel tramadol lotions. Tropical Journal of Pharmaceutical Research, 12, 27-32, 2013.
[12] Williams A.C., Barry B.W. Penetration enhancers. Advanced Drug Delivery Reviews, 56, 603-618, 2004.

[13] Som I., Bhatia K., Yasir M. Status of surfactants as penetration enhancers in transdermal drug delivery. Journal of Pharmacy and Bioallied Sciences, 4, 2-9, 2012.

[14] Shahzad Y., Afreen U., Shah S.N.H., Hussain T. Applying response surface methodology to optimize nimesulide permeation from topical formulation. Pharmaceutical Development and Technology, 18, 1391-1398, 2013.

[15] Javadzadeh Y., Shokri J., Hallaj-Nezhadi S., Hamishehkar H., Nokhodchi A. Enhancement of percutaneous absorption of Finasteride by cosolvents, cosurfactant and surfactants. Pharmaceutical Development and Technology, 15, 619-625, 2010.

[16] Zhu X., Ji J., Huang D., Zhu Y., Tang C., Yang X. Discovery, Synthesis and Evaluation of Novel Cholesterol Absorption Inhibitors. Chemical Biology \& Drug Design, 80, 426-433, 2012.

[17] Shah S.N.H., Hussain T., Khan I.U., Asghar S., Shahzad Y. Formulation study of topically applied lotion: in vitro and in vivo evaluation. Bioimpacts, 3, 11-99, 2013.

[18] Chang J.S., Wu P.C., Huang Y.B., Tsai Y.H. In-vitro evaluation of meloxicam permeation using response surface methodology. Journal of Food and Drug Analysis, 14, 236-241, 2006.

[19] Megrab N.A., Williams A., Barry B. Oestradiol permeation through human skin and silastic membrane: effects of propylene glycol and supersaturation. Journal of Controlled Release, 36, 277-294, 1995.

[20] Marrakchi S., Maibach H.I. Sodium lauryl sulfate-induced irritation in the human face: Regional and age-related differences. Skin Pharmacology and Physiology, 19, 177-180, 2006.

[21] Molgaard B., Hoelgaard A. Vehicle effect on topical drug delivery. I. Influence of glycols and drug concentration on skin transport. Acta Pharmaceutica Suecica, 20, 433-442, 1983.

[22] Barry B.W. Mode of action of penetration enhancers in human skin. Journal of Controlled Release, 6, 85-97, 1987.

[23] Kaushik D., Costache A., Michniak-Kohn B. Percutaneous penetration modifiers and formulation effects. International Journal of Pharmaceutics, 386, 42-51, 2010.

[24] Waters L.J., Dennis L., Bibi A., Mitchell J.C. Surfactant and temperature effects on paraben transport through silicone membranes. Colloids and Surfaces B: Biointerfaces.108, 23-28, 2013. 